\title{
A STUDY TO COMPARE THE EFFICACY OF ULTRASOUND WITH ECCENTRIC EXERCISES AND ULTRASOUND WITH CONCENTRIC EXERCISES ON TENDO ACHILLES TENDINITIS IN ATHELETES
}

\author{
Ravish V. N¹, R. Raja로 S. R. Sarala ${ }^{3}$, Pavan Vivek Raj ${ }^{4}$
}

HOW TO CITE THIS ARTICLE:

Ravish V. N, R. Raja, S. R. Sarala, Pavan Vivek Raj. "A Study to Compare the Efficacy of Ultrasound with Eccentric Exercises and Ultrasound with Concentric Exercises on Tendo Achilles Tendinitis in Athletes". Journal of Evolution of Medical and Dental Sciences 2014; Vol. 3, Issue 09, March 03; Page: 2258-2267,

DOI: $10.14260 /$ jemds/2014/2136

ABSTRACT: In a previous uncontrolled pilot study we demonstrated very good clinical results with eccentric calf muscle training on patients with painful chronic Achilles tendinosis located at the 2-6 $\mathrm{cm}$ level in the tendon. In the present prospective study patients with painful chronic Achilles tendinosis at the 2-6 cm level in the tendon were randomized to treatment with either an eccentric or a concentric training regimen for the calf muscles with therapeutic ultrasound. The study included 60 patients, with 30 in each group mean age 30 years in each treatment group. The amount of pain during activity (jogging or walking) was recorded by the patients on a visual analogue scale, and patient satisfaction was assessed before and after treatment. The patients were instructed to perform their eccentric or concentric training regimen on a daily basis for 12 weeks. In both types of treatment regimen the patients were told to do their exercises despite experiencing pain or discomfort in the tendon during exercise. The results showed that after the eccentric training regimen $80 \%$ of the patients (24/30) were satisfied and had resumed their previous activity level (before injury), compared to $63 \%$ of the patients $(19 / 30)$ who were treated with the concentric training regimen with therapeutic ultrasound as the common modality. The results of means of pain is (0.902) is significant, for range of motion is (0.042) which is not significant and foot ankle ability measure is (0.311) is significant after treatment with eccentric training was significantly better than after concentric training.

KEYWORDS: Achilles tendinitis, eccentric exercises, concentric exercises, therapeutic ultrasound.

INTRODUCTION: A chronic painful condition in Achilles tendon is relatively common, especially in middle-aged male runners. ${ }^{1,2}$ The nomenclature for these conditions is confusing and often does not reflect the pathology of the tendon disorder. The term tendinosis has become widely accepted in recent literature on patients with a chronic painful condition in the middle portion of the tendon and where radiographic images or ultrasonography shows a tendon disorder. ${ }^{3}$ The characteristic morphological features of tendinosis have been demonstrated to be an increased amount of interfibrillar glycosaminoglycans and changes in the collagen fiber structure and arrangement 4,5 . The cause and pathogenesis are unknown. It is considered difficult to treat this condition, but a recent study reported very good results with heavy-load eccentric calf muscle training on patients with Achilles tendinosis located at the 2-6 cm level (from the calcaneal insertion) in the tendon 1. However, that was a pilot study evaluating the effect of eccentric training and not a randomized study comparing the effects of different treatment regimens. The aim with this study was prospectively to compare two different treatment models (eccentric versus concentric calf muscle training) in a randomized manner on patients with painful Achilles tendon. 
MATERIAL AND METHODS: This study included 60 patients with severe Achilles tendon pain and who had been referred to us as candidates for surgical treatment The study protocol was approved by the ethics committee of the Kempegowda institute of medical sciences (KIMS)Medical Faculty, Patients were randomized to treatment either with eccentric calf muscle training or with concentric calf muscle training. Most of the patients had a long duration of symptoms. The patient was in prone position with both feet free of the examination table, and the Achilles tendons were examined in the longitudinal and transverse plane. In all patients a painful area of the tendon was found at the 2-6 cm level (from the calcaneal insertion) in the tendon.

RANDOMIZATION PROCEDURE: The patients were examined by the orthopedic surgeon. Patients with restricted ankle joint motion due to other injuries or diseases were not included. If the results of clinical examination (painful tendinosis at the $2-6 \mathrm{~cm}$ level in the tendon), the patient was carefully informed and asked about participation in the study. The patients picked an envelope containing a paper allocating the patient to either an eccentric or a concentric training regimen. Thirty patients group A (30 men) with a mean age of 24.167 years eccentric training group, and group B 30 patients(30 men) with a mean age of 24.667 years (range $34-61$ ) were randomized to the concentric training group. There were no significant differences in gender, age, weight, height, or duration of symptoms between the two groups of patients.

TREATMENT MODELS: There were two different types of treatment model, eccentric calf muscle training and concentric calf muscle training with therapeutic ultrasound. The eccentric training model was exactly the same that was used in the recently published article on eccentric calf muscle training on patients with chronic Achilles tendinosis 6. The concentric training model was constructed and emphasis was placed on the purpose that it should include the most commonly used exercises for concentric calf muscle training. Great care was taken to give exactly the same instructions. All patients were instructed on the way in which to perform their training regimen.

ECCENTRIC TRAINING REGIMEN: The patients were instructed to do their eccentric exercises, 7 days/week, for 12 weeks. During the 12-week training regimen jogging/walking activity was allowed if it could be performed with only mild discomfort and no pain. The patients were instructed to start jogging or walking activity at a slow pace, on flat ground, and for a short distance. Thereafter their activity could be gradually increased if there was no severe pain in the tendon. ${ }^{7}$

ECCENTRIC EXERCISES: Two types of exercises were used (Figs. 1, 2). The calf muscle was eccentrically loaded both with the knee straight (Fig. 1) and to maximize the activation of the soleus muscle also with the knee bent (Fig. 2). Each of the two exercises included 15 repetitions in three sets $(3 \times 15$ repetitions). The patients were told that muscle soreness during the first 1-2 weeks of training was to be expected. In the beginning the loading consisted of the bodyweight, and the patients were standing with all their body weight on their injured leg (lifted to that position by the uninjured leg). From an upright body position and standing with all body weight on the forefoot, with the ankle joint in plantar flexion, the calf muscle was loaded by having the patient to lower the heel beneath the lever (Figs. 1,2). They loaded the calf muscle only eccentrically; no concentric loading followed. Instead, the noninjured leg was used to return to the starting position. The patients 
were told to continue doing the exercise despite experiencing pain during the exercise. However, no training through disabling pain was encouraged. When they were able to perform the eccentric loading without experiencing any minor pain or discomfort, they were instructed to increase the load by adding weight. This could be done easily using a backpack that was successively loaded with weight (Fig. 3). This gradually increased the eccentric calf muscle loading. When very heavy weights were needed, the patients were told to use a weight machine (Fig. 4) following which ultrasound was given with $1.0 \mathrm{~W} / \mathrm{cm}^{2}$ for $4 \mathrm{~min}(\mathrm{Fig} 5)^{7}$

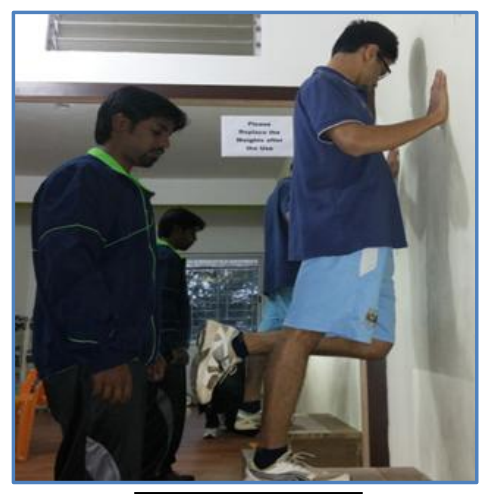

Fig. 1

Fig. 1: Eccentric calf-muscle loading with the knee straight. From an upright body position and standing with all body weight on the ventral half-part of the foot, with the ankle joint in plantar flexion lifted by the noninjured leg, the calf-muscle was loaded eccentrically by having the patient to lower the heel beneath the lever.

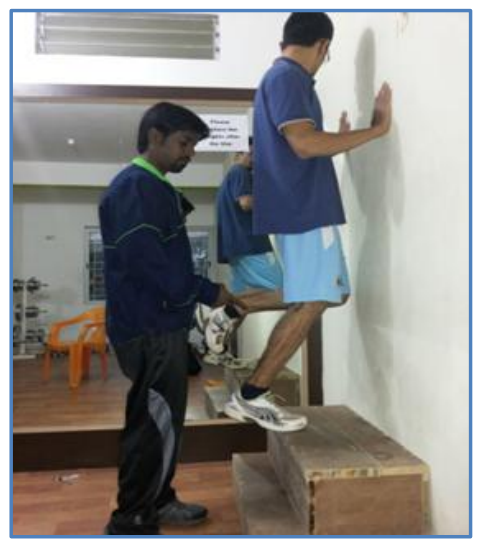

Fig. 2

Fig. 2: Eccentric calf-muscle loading with the knee bent. From an upright body position and standing with all body weight on the ventral half-part of the foot, with the ankle joint in plantar flexion lifted by the noninjured leg, the calf-muscle was loaded eccentrically by having the patient to lower the heel beneath the lever. 


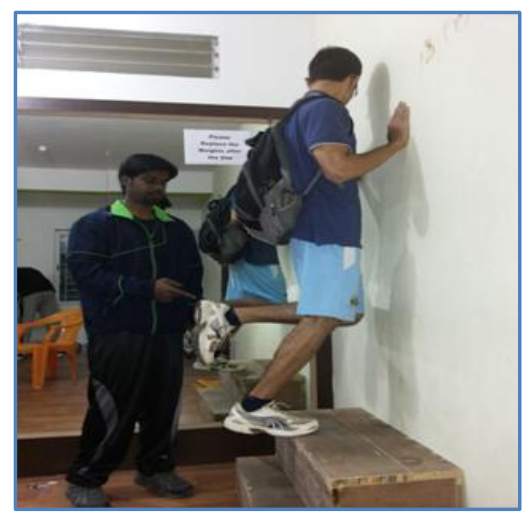

Fig. 3: Elevating the load by adding weight in a back-pack.

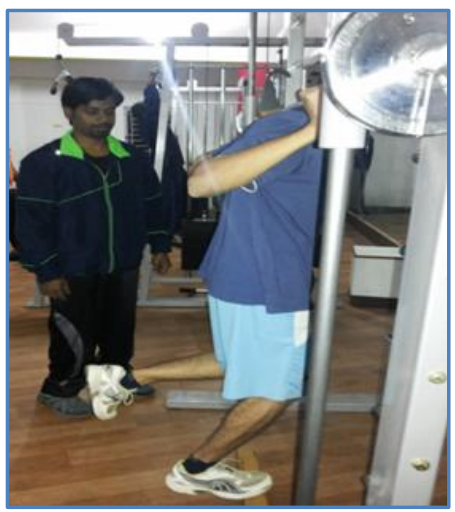

Fig. 4: Elevating the load by adding weight in a weight machine

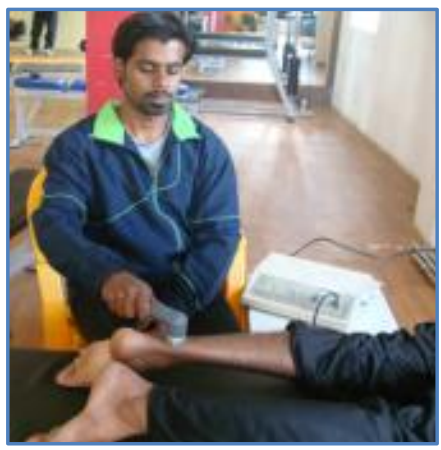

Fig. 5: Therapeutic ultrasound

CONCENTRIC TRAINING REGIMEN: The patients were instructed to carry out their concentric exercises 7 days/week, for 12 weeks. During the 12-week training regimen, running or walking activity was allowed if it could be performed with only mild discomfort and no pain. The patients were instructed to start jogging or walking activity at a slow pace, on flat ground, and for a short distance. Thereafter their activity could be gradually increased if no severe pain in the tendon ${ }^{7}$

\section{CONCENTRIC EXERCISES:}

Weeks 1-2: Two types of exercises were used (Figs. 6, 7). The calf muscle was concentrically loaded both with the knee straight (Fig. 6) and to maximize the activation of the soleus muscle also with the knee bent (Fig. 7). Each of the two exercises included 20 repetitions carried out in two or three sets $(2 / 3 \times 15$ repetitions). The patients were told to continue doing the exercise despite experiencing pain during the exercise. However, no training through disabling pain was encouraged. The patients were told that muscle soreness during the first 1-2 weeks of training might be expected. ${ }^{7}$

Weeks 3-5: Two types of exercises were used (Figs. 8,9). The calf muscle was concentrically loaded with the knee straight (Fig. 8; $3 \times 15$ repetitions). The patients stood with all their bodyweight on their injured leg, and the heel was lifted from the ground by concentric contraction of the calf muscle. They loaded the calf muscle only concentrically; no eccentric loading followed. Instead, the 


\section{ORIGINAL ARTICLE}

noninjured leg was used to return to the starting position. To maximize the activation of the soleus muscle, step-ups were used with the knee bent (Fig. 8; $3 \times 1 \mathrm{~min}$, slow speed) was used.

Weeks 6-12: Four types of exercises were used (Figs. 8, 9, 10,11). The calf muscle was concentrically loaded both with the knee straight (Fig. 8; $3 \times 15$ repetitions)and to maximize the activation of the soleus muscle also with the knee bent (Fig. 9; $3 \times 1 \mathrm{~min}$, slow speed). Two exercises, rope skipping (Fig. 10; 3-4 min, slow speed) and side jumps (Fig. 11; $3 \times 20$ repetitions), were used with the purpose of placing heavy loads on the calf muscle. ${ }^{7}$

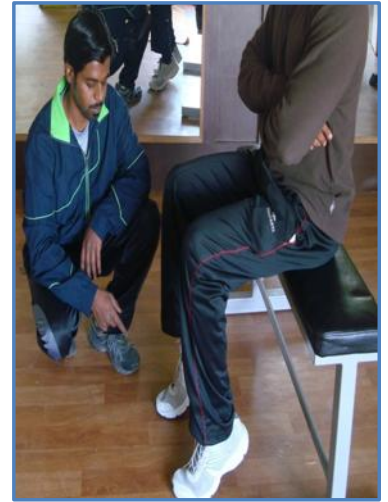

Fig. 6: Concentric loading of the calf-muscle with the knee straight

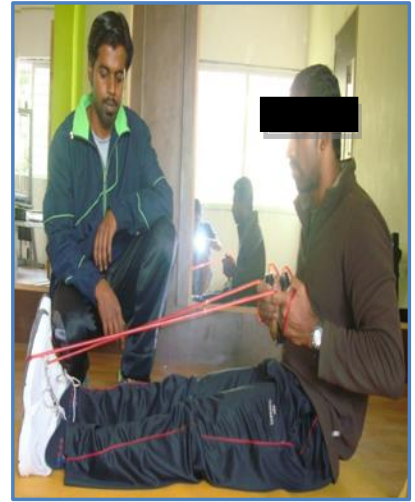

Fig. 7: Concentric loading of the calf-muscle with the knee bent

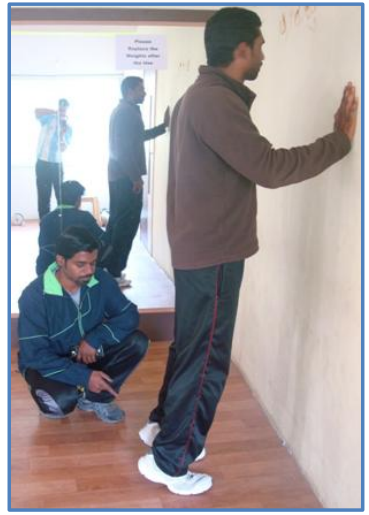

Fig. 8: Heel raises from an upright body position, with the knee straight and standing with all body weight on the injured side

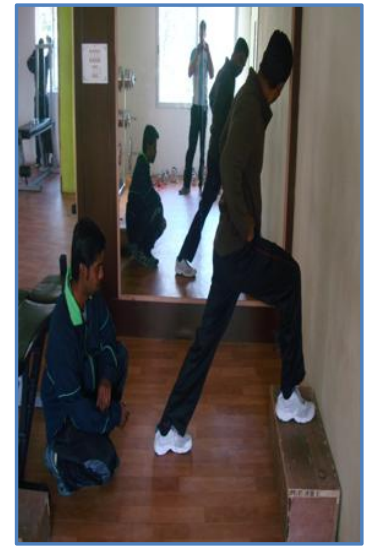

Fig. 9: Step ups on a bench, with the knee bent and with all body weight on the injured side 

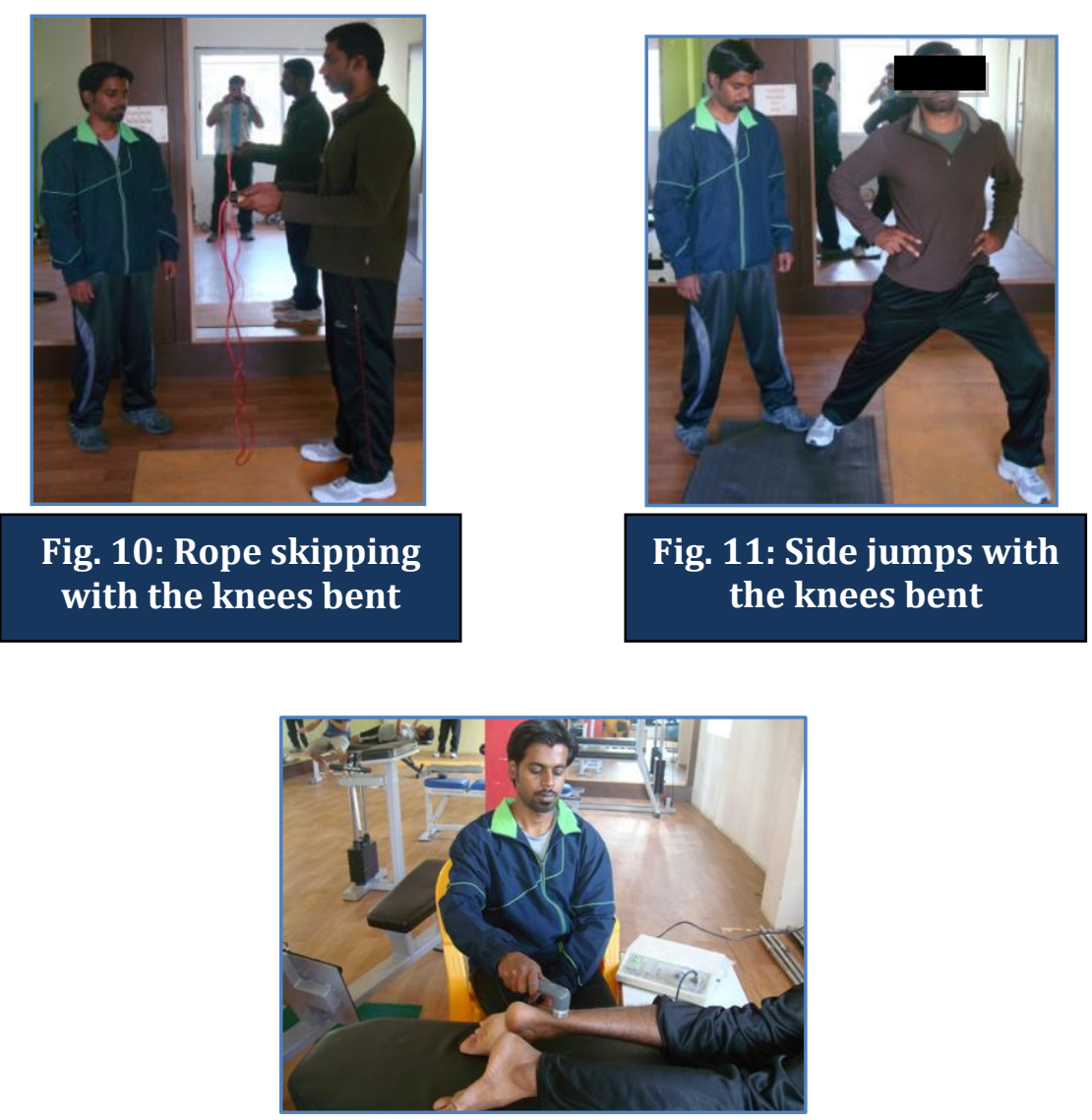

Fig. 12: Therapeutic ultrasound

EVALUATION: Results were evaluated with the use of a visual analogue scale (VAS) for pain, where the patient recorded the amount of pain during activity (running or walking) on a 10-cm-long pain scale. The amount of pain was recorded from $0-10 \mathrm{~cm}$, where no pain is recorded as 0 and severe pain as 10 . The purpose of goniometry is to measure the angle of joint position or range of joint motion. Therefore, a valid goniometric measurement is one that truly represents the actual joint angle or the total Range of Motion. The ankle range of motion can be measured by the way of keeping fulcrum at the lateral malleoli, the mobile arm should be fixed at the lateral border of the foot and the stable arm should be placed parallel to the fibula for recording the ankle Range of Motion Foot and Ankle Ability Measure (FAAM) is a self-reported tool of physical function for individuals with leg, ankle, and foot musculoskeletal disorders. The tool consists of the 21-item activities of daily living (ADL) and 8-item Sports subscales, which together produced information about the sample Patient satisfaction was also assessed. Results were evaluated before (week 0) and after (week 12) treatment. Data are presented as means $\pm S D$. Student ' $t$ ' test was used to compare the results after treatment with eccentric and concentric training. A P value lower than 0.05 was considered significant.

THERAPEUTIC ULTRASOUND: Therapeutic ultrasound reduces the swelling in the acute inflammatory phase and improves healing of the tendon. It also stimulates the synthesis of collagen 
in tendon fibroblasts and cell division during periods of rapid cell proliferation. Therapeutic ultrasound is hence a desirable treatment option in the treatment of acute Achilles tendinopathy. Further there are so many researchers used ultrasound as the ideal modality for treating Achilles tendinopathy.

Both the groups are administered ultrasound in the direct contact technique with:

- $1 \mathrm{mhz}$ ultrasound modality

- 0.8-1.0watts $/ \mathrm{cm}^{2}$ intensity for 6 minutes.

- Ultrasonic gel is used as a coupling media.

- $2 \mathrm{cms}$ Diameter transducer.

The subjects are assessed with VAS, FAAM and Ankle Range of motion with goniometer at the end of $1^{\text {st }}, 2^{\text {nd }}$ and $3^{\text {rd }}$ month respectively.

RESULTS: Before treatment (week 0) all patients had pain during activity (jogging/walking). In the group treated with the eccentric training regimen $80 \%(24 / 30)$ of the patients were satisfied and resumed their previous activity level (before injury) after 12 weeks of training. In the group treated with the concentric training regimen 63\% (19/30) were satisfied and resumed their previous activity level (before injury) after 12 weeks of training. The results of treatment with the eccentric training regimen was significantly better $(\mathrm{P}<0.05)$ than the results of treatment with the concentric training regimen. The mean VAS score in the 24 patients treated with eccentric training who were satisfied with treatment decreased. In the group treated with concentric training there were 19 who were satisfied with treatment, and their mean VAS score decreased significantly from after treatment. The range of motion of the in both the groups which were treated with eccentric training regime and concentric training regime did not show significant improvement. Foot Ankle Ability measure Activities of Daily Living (ADL) subscale contained 26 items pertaining to basic functional activities and pain. The Sports subscale contained eight items pertaining to higher level activities, such as those required in athletics. Where 24 out of 30 patients from group B showed improvement in the performance of the activities of daily living and sporting activities.

DISCUSSION: In this randomized prospective study comparing two different treatment models on patients with Achilles tendinosis we reproduced the good results that had previously been shown with the eccentric training model (but without using a randomized concentric training group for comparison) ${ }^{8}$. We also found that the eccentric training regimen produced significantly better results than a concentric training regimen. The cause of chronic Achilles tendinosis is unknown, but the condition is generally associated with over use from repetitive loading 9,10,11. However, this condition is also seen in individuals who are not physically active, and found no correlation between physical activity and histopathology in a large group of patients with Achilles tendinopathy. Tendinosis really is a condition that is associated with overuse.

However, for individuals with a very sedentary life-style, short walks may be enough activity to cause overuse symptoms from the Achilles tendons. Achilles tendinosis is most often, but not always, associated with pain. The patients in this study all had pain during activity (jogging or running). The pain is often treated by nonsteroidal anti-inflammatory drugs (NSAIDs) ${ }^{12}$, despite the 
fact that biopsy studies repeatedly show an absence of inflammatory cells 13,14 , and that a randomized placebo-controlled study using piroxicam has shown similar results as with placebo ${ }^{15}$. In our study $61 \%$ of the patients had been treated with NSAIDs. The background to the association with pain is unknown, but in a recent investigation using a microdialysis technique we observed greater amounts of the excitatory neurotransmitter glutamate in Achilles tendons with tendinosis than in normal tendons 16 . That investigation also demonstrated no signs of chemical inflammation in tendons with tendinosis (normal prostaglandin $\mathrm{E}_{2}$ levels).

Treatment of chronic Achilles tendinosis generally consists of a combination of different "attack points." Many different designs of combined rehabilitation models have been proposed, most of which include a combination of rest (complete or modified), orthotic treatment (heel lift, change of shoes, corrections of mal-alignment), medication (NSAIDs, corticosteroids, cold therapy), stretching and massage, and strength training. ${ }^{17,18,19}$ It is important to note that there is only sparse scientific evidence for most of the conservative treatments proposed and used in patients with painful conditions in the Achilles tendon.

There are few scientific prospective studies, and the absence of studies comparing different types of conservative treatment regimens in a randomized manner is a major disadvantage when evaluating the effects of the specific treatment regimens. There has been no treatment of choice for this condition, but in a recent pilot study treatment with eccentric calf muscle training demonstrated very good clinical results on patients with chronic Achilles tendinosis located at the 2-6 cm level in the tendon. However, that study included no comparison with other types of treatment modalities.

In our study the clinical examination revealed a painful area at the 2-6 cm level in the tendon, The two training groups in our study were comparable in respect of the duration of symptoms and the clinical diagnosis Influenced by the theories of Curwin and Stanish on treatment of tendinitis with eccentric exercises, we earlier designed an eccentric calf muscle training regimen that yielded very good results in a pilot study of 15 patients with painful chronic Achilles tendinosis at the 2-6 cm level in the tendon. The eccentric training regimen used in this study contained exactly the same type of eccentric exercises as in our previous study. The concentric exercises were carefully selected and were to consist mainly of concentric muscle activation. However, in some of the exercises there is also some eccentric loading.

In both the eccentric and concentric training regimen the patients were instructed to perform their exercises despite experiencing pain in the tendon when performing the exercise. Therefore, and this needs to be emphasized, in both types of treatment regimen the patients were training despite having pain in the tendon during exercise. The patients were instructed to carry out their exercises daily for 12 weeks. We used no other tools to control compliance because we believed that the patients were sufficiently motivated to perform their treatment regimen although they all had had a long duration of symptoms, and the alternative treatment was surgery.

We cannot explain why the eccentric training regimen produced significantly better results than the concentric regimen. Theoretically, it may have been an effect of increased eccentric calf muscle strength, or caused by a lengthening of the muscle-tendon unit and consequently less load on the tendon during motion. Also, this type of loading (eccentric) may possibly be associated with changes in the metabolism of certain substances in the tendon causing alterations in the pain perception from the tendon. 
In conclusion, treatment with eccentric calf muscle training in patients with painful Achilles tendinosis yielded good short-term clinical results and significantly better results than were achieved with concentric calf muscle training treatment models. Before resorting to surgical treatment, we recommend therapeutic ultrasound and eccentric calf muscle training in all patients with painful Achilles tendinosis located at the 2-6 cm level in the tendon.

\section{REFERENCES:}

1. Sandmeier R, Renström PAFH. Diagnosis and treatment of chronic tendon disorders in sports. (1997) Cand J Med Sci Sports 7:96-106.

2. Welsh RP, Clodman J. Clinical survey of Achilles tendinitis in athletes. (1980) Can Med Assoc J 122:193-195

3. Khan KM, Bonar F, Desmond PM et al. Patellar tendinosis (jumper's knee): findings at histopathologic examination, US, and MR imaging. Victorian Institute of Sport tendon Study Group. (1996) Radiology 200:821-827.

4. Rolf C, Movin T. Etiology, histopathology and outcome of surgery in achillodynia. Foot Ankle Int1997;18:565-9.

5. Khan KM, Cook JL, Bonar F, Harcourt P, Åström M Histopathology of common tendinopathies. Update and implications for clinical management. (1999)Sports Med 27:393-408.

6. Alfredson H, Pietilä T, Öhberg L et al. Achilles tendinosis and calf muscle strength. The effect of short-term immobilization after surgical treatment.(1998a) Am J Sports Med 26:166-171.

7. N. Mafi R. Lorentzon H. Alfredson. Superior short-term results with eccentric calf muscle training compared to concentric training in a randomized prospective multicenter study on patients with chronic Achilles tendinosis Knee Surg, Sports Traumatol, (2001) Arthrosc (2001) $9: 42-47$.

8. Alfredson $\mathrm{H}$, Pietilä $\mathrm{T}$, Jonsson $\mathrm{P}$ et al. Heavy-load eccentric calf muscle training for the treatment of chronic Achilles tendinosis. Am J (1998b).

9. Józsa L, Kannus P. Human tendons. Anatomy, physiology, and pathology. Human Kinetics, Champaign (1997).

10. Archambault JM, Wiley P, Bray RC. Exercise loading of tendons and the development of overuse injuries: a review. (1995) Sports Med 20:77-89.

11. Curwin S, Stanish WD. Tendinitis: its etiology and treatment. Collamore, Lexington (1984).

12. Khan KM, Bonar F, Desmond PM et al. Patellar tendinosis (jumper's knee): findings at histopathologic examination, US, and MR imaging. Victorian Institute of Sport tendon Study Group. (1996) Radiology 200:821-827.

13. Movin T (1998) Aspects of aetiology, pathoanatomy and diagnostic methods in chronic midportion Achillodynia. Thesis, Karolinska Institute Stockholm.

14. Åström M (1997) On the nature and etiology of chronic Achilles tendinopathy Thesis, University of Lund.

15. Åström M, Westlin N. No effect of piroxicam on Achilles tendinopathy: a randomized study of 70 patients. (1992)Acta Orthop Scand 63:631-634.

16. Alfredson $\mathrm{H}$, Thorsen $\mathrm{K}$, Lorentzon $\mathrm{R}$ In situ microdialysis in tendon tissue: high levels of glutamate, but not prostaglandin $\mathrm{E}_{2}$ in chronic Achilles tendon pain. Knee Surg Sports Traumatol (1999) Arthrosc 7:378-381. 


\section{ORIGINAL ARTICLE}

17. Welsh RP, Clodman J Clinical survey of Achilles tendinitis in athletes. (1980) Can Med Assoc J 122:193-195.

18. Galloway MT, Jokl P, Dayton OW Achilles tendon overuse injuries. (1992) Clin Sports Med 11:771-782.

19. Józsa L, Kannus P (1997) Human tendons. Anatomy, physiology, and pathology. Human Kinetics, Champaign.

\section{AUTHORS:}

1. Ravish V. N

2. R. Raja

3. S. R. Sarala

4. Pavan Vivek Raj

\section{PARTICULARS OF CONTRIBUTORS:}

1. Associate Professor, Department of Orthopaedics, Kempegowda Institute of Medical Sciences, Bangalore.

2. Associate Professor, Department of Physiotherapy (Musculo-Skeletal disorders and Sports Medicine), KIPT, Bangalore.

3. Senior Scientific Officer, Department of Sports Medicine, Sports Authority of India, Bangalore.
4. Post Graduate, Department of Physiotherapy KIPT, Bangalore.

\section{NAME ADDRESS EMAIL ID OF THE} CORRESPONDING AUTHOR:

Dr. PavanVivek Raj,

P. V. Crib, \#9/2, $2^{\text {nd }}$ Cross,

Tavarekere, Bangalore - 560029 .

E-mail: pavan988031@gmail.com

Date of Submission: 24/01/2014.

Date of Peer Review: 25/01/2014.

Date of Acceptance: 07/02/2014.

Date of Publishing: 28/02/2014. 